P0129

\title{
EXPERIMENTAL RESEARCH OF DYNAMIC LIGHTING SCHEMES ON IMPROVING THE NEGATIVE EMOTION OF LOW VISION PATIENTS
}

\author{
Luoxi Hao et al. \\ DOI 10.25039/x46.2019.PO129 \\ from \\ CIE x046:2019 \\ Proceedings \\ of the \\ 29th CIE SESSION \\ Washington D.C., USA, June 14 - 22, 2019 \\ (DOI 10.25039/x46.2019)
}

The paper has been presented at the 29th CIE Session, Washington D.C., USA, June 14-22, 2019. It has not been peer-reviewed by CIE.

(C) CIE 2019

All rights reserved. Unless otherwise specified, no part of this publication may be reproduced or utilized in any form or by any means, electronic or mechanical, including photocopying and microfilm, without permission in writing from CIE Central Bureau at the address below. Any mention of organizations or products does not imply endorsement by the CIE.

This paper is made available open access for individual use. However, in all other cases all rights are reserved unless explicit permission is sought from and given by the $\mathrm{CIE}$.

CIE Central Bureau

Babenbergerstrasse 9

A-1010 Vienna

Austria

Tel.: +4317143187

e-mail: ciecb@cie.co.at

www.cie.co.at 


\title{
EXPERIMENTAL RESEARCH OF DYNAMIC LIGHTING SCHEMES ON IMPROVING THE NEGATIVE EMOTION OF LOW VISION PATIENTS
}

\author{
Hao, L.X. ${ }^{1,2}$, Wang, T.Y..$^{1,2}$ \\ ${ }^{1}$ College of Architecture and Urban Planning, Tongji University, Shanghai, CHINA, \\ ${ }^{2}$ Key Laboratory of Ecology and Energy-saving Study of Dense Habitat (Tongji University), \\ Ministry of Education, Shanghai, CHINA \\ haoluoxi@tongji.edu.cn
}

DOI 10.25039/x46.2019.PO129

\begin{abstract}
By changing the brightness of functional lighting and the colour of ambient lighting, the facial expressions of patients with low vision were monitored and the changes of their negative emotions were analysed. In the day ward of the eye hospital, 16 patients were randomly selected as subjects and divided into 4 groups on average. One group was the reference group with only functional lighting, and the other three were the intervention group with additional coloured ambient light. The results showed that the gender difference was not significant, the negative emotions in the reference group were significantly higher than those in the experimental group, and the improvement effect of the emotional lights in the purple, orange and blue groups was significant, among which the orange group had the best effect, while the blue group and the purple group had better effect, but the difference was not significant.
\end{abstract}

Keywords: Iow vision, LED, emotion regulation, colour, expression analysis

\section{Introduction}

According to the statistics of the World Health Organization (WHO), there are a total of 246 million people with low vision all over the world, including 67.3 million Chinese (CIE 2017). The diagnostic criteria for the patients with low vision eyes are the best corrected visual acuity of $0.05 \sim 0.3$, and sight radius of more than $10^{\circ}$ and less than $15^{\circ}$. People used to ignore low vision because they thought they still could "see". The main causes of low vision include hereditary congenital eye disease, amblyopia, high myopia, corneal leukoplakia, glaucoma, cataract and aphakia, diabetic fundus changes and retinal pigmentation (Cao et al. 2009). Limited vision tends to cause psychological problems of patients, bring anxiety, depression and other negative emotions, and further reduce the quality of life (Duan. 2018). Psychological nursing, social attention and support are often adopted in clinical practice to help patients to reduce psychological stress, but more medical work and social support are needed (Li et al. 2016) (Liu. 2016).

With the discovery of ipRGCs, the non-visual photoreceptor cells on the retina, the non-visual effect mechanism of lighting has gradually become clear. It provides a theoretical basis for the application of healthy lighting from meeting the needs of visual work to emotional regulation, sleep quality, environmental cognition, rhythm repair and other aspects (Hao et al. 2015). Based on the study about the influence of light colour and dynamic lighting on atmosphere perception and physiological relaxation, Ham et al. (2012) found that the slowly changing orange light environment could create a relaxed and comfortable atmosphere. According to years of clinical nursing experience, Hatfield (2013) pointed out that a soft dark light environment could help parturients to relieve tension and achieve better delivery effect. Figueiro et al. (2015) conducted a lighting experiment on the patients with Alzheimer's disease for 11 weeks, and found that the lighting environment set in the experiment effectively improved the sleep efficiency of the subjects and alleviated their depression. Studies show that the ones with severe depression have weakened facial reactions to positive stimulus. Reed et al. (2007) tested the comic effect on depression in patients with facial expressions through facial expression analysis method, and found that people with symptoms of depression were more likely to control their original smile and negative related expression than people without symptoms of depression. Zhang et al. (2018) attempted to apply the facial expression analysis method to evaluate the influence of 
tunnel lighting environment on driving nervousness, so as to obtain the optimal ratio of road brightness and environment brightness in the tunnel.

Patients with low vision are more likely to have symptoms such as blurred vision, decreased vision and eye pain. They are prone to produce anxiety, fear, tension and depression, and fear of seeing light. Thus, strong light stimulation should be avoided in clinical treatment. The daytime ward of the eye hospital is a diagnosis and treatment unit area set for patients with low vision who seek for diagnosis and treatment, hospitalize for short-term ophthalmic observation and receive special examination. It is also the main area for preoperative waiting and postoperative recuperation of patients with low vision. Patients' negative emotions are more obvious. Thus, the lighting environment of the ward on the spot is changed, and mood lighting lamps of different colours are set. Besides, the background brightness of functional lighting is adjusted based on the diurnal variation law of natural light. The negative mood changes of patients with low vision are monitored by the facial expression analysis so as to obtain appropriate lighting mode.

\section{Methods}

\subsection{Subjects}

The subjects were patients in the day ward of an eye hospital. 16 patients were randomly selected as subjects, and they were divided into 4 groups on average, half male and half female. One group was the reference group and the other three were the intervention group. All subjects signed the informed consent approved by their institutional review committee.

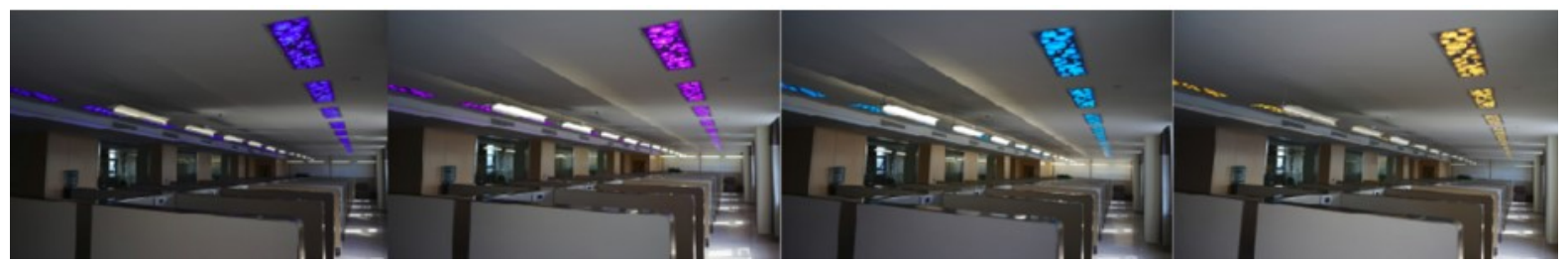

Figure 1 - Four light patterns in the day ward

\subsection{Experimental scenes}

It was done in the day ward of an eye hospital. The preset four modes of examination and treatment (cyan), decompression and relaxation (purple), postoperative recuperation (orange) and dark light repose (blue) were used to change the indoor ambient brightness and the colour of mood panel light (see figure 1 and 2). The examination and treatment mode is rarely used because the brightness is too high, which is generally unacceptable to patients. In the morning, when the patient is waiting for surgery in the daytime ward, the light environment with high physiological stimulation value (CS) should be adopted. At noon, with the enhancement of sunlight, the indoor illumination should not be too high, so the postoperative recuperation mode with moderate brightness was adopted. In the afternoon, the patient gradually returned to the ward after the operation and rested in the dark light mode with low brightness. In the morning and afternoon, two subjects were selected as the reference group, and the mood panel light above the corresponding position was turned off, leaving only functional lighting.

The hand-held spectrophotometer CL-500A produced by Konica company was used to calibrate the illumination and colour temperature of patients' eyes when they recline to rest (see table 1), and the spectral power distribution under different light modes was measured (see figure 3 ). 


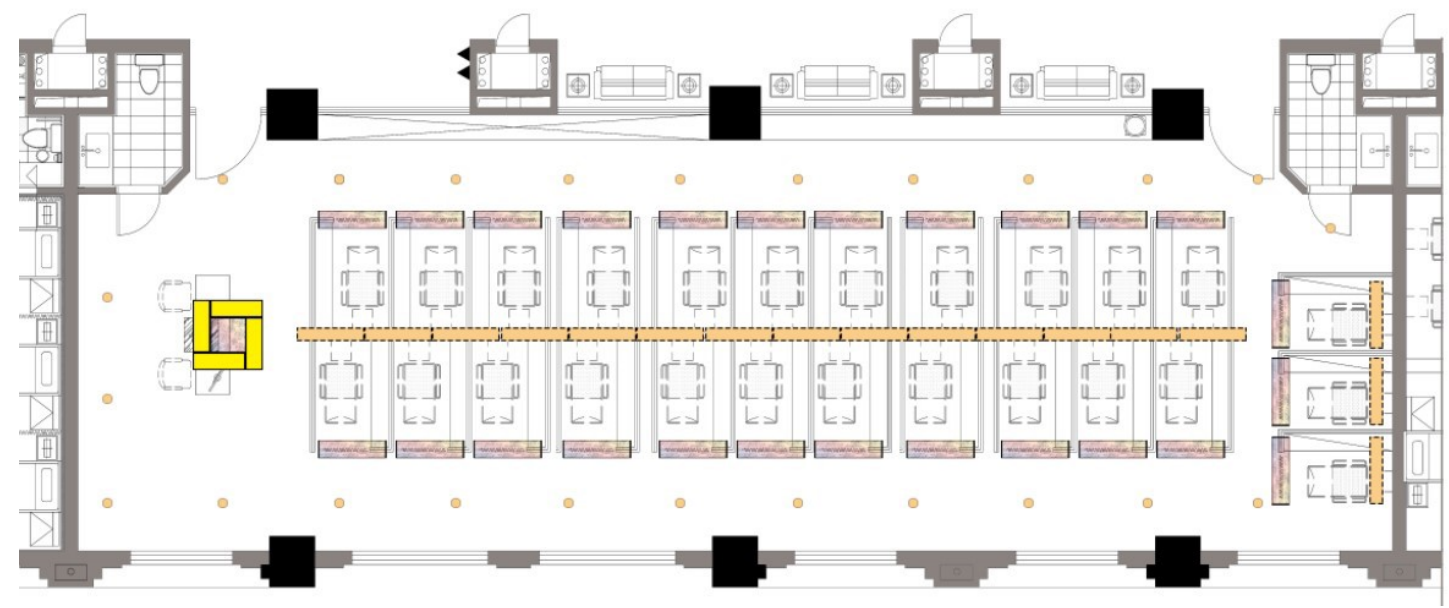

Figure 2 - Floor plan and lighting arrangement of day ward

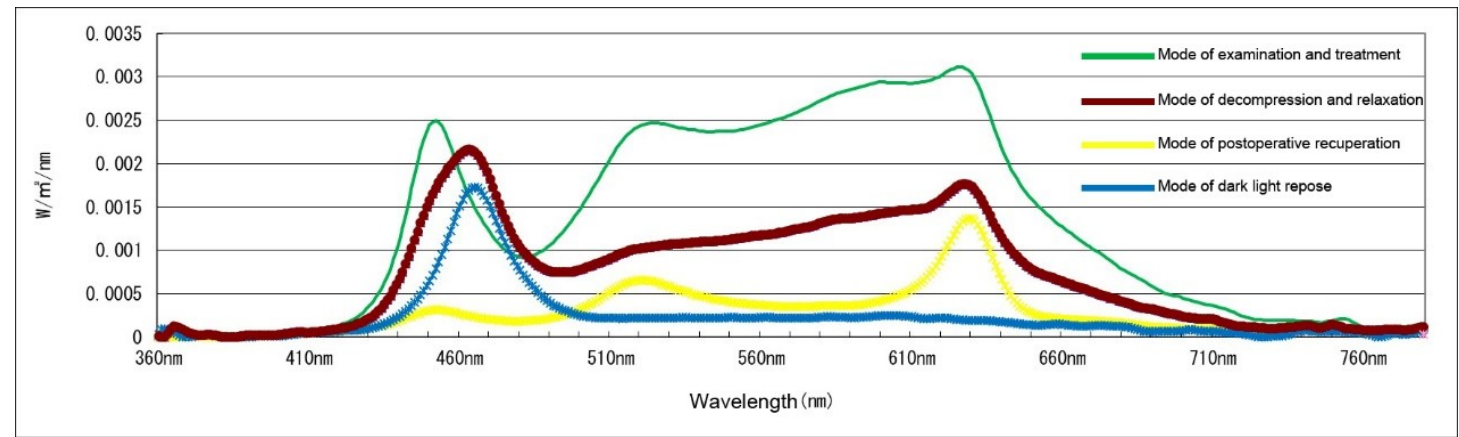

Figure 3 - Spectral power distribution of human eyes in different scene modes

Table 1 - Light parameters of human eyes in different scene modes

\begin{tabular}{|l|l|l|l|l|l|l|}
\hline & \multicolumn{2}{|l|}{$\begin{array}{l}\text { Percentage of } \\
\text { panel } \\
\text { power }\end{array}$} & $\begin{array}{l}\text { lamp } \\
\text { lamp }\end{array}$ & $\begin{array}{l}\text { Eye } \\
\text { illumination } \\
\text { /lx }\end{array}$ & CCT/K & $\begin{array}{l}\text { Peak } \\
\text { wavelength } \\
\text { /nm }\end{array}$ \\
\hline $\begin{array}{l}\text { Mode of examination } \\
\text { and treatment }\end{array}$ & $4000 \mathrm{~K}$ & $100 \%$ & cyan & 177.99 & 3858 & 626 \\
\hline $\begin{array}{l}\text { Mode of decompression } \\
\text { and relaxation }\end{array}$ & $4000 \mathrm{~K}$ & $80 \%$ & purple & 87.41 & 4495 & 463 \\
\hline $\begin{array}{l}\text { Mode of postoperative } \\
\text { recuperation }\end{array}$ & $4000 \mathrm{~K}$ & $40 \%$ & orange & 34.39 & 3492 & 629 \\
\hline $\begin{array}{l}\text { Mode of dark light } \\
\text { repose }\end{array}$ & $4000 \mathrm{~K}$ & $10 \%$ & blue & 18.44 & 7234 & 465 \\
\hline
\end{tabular}




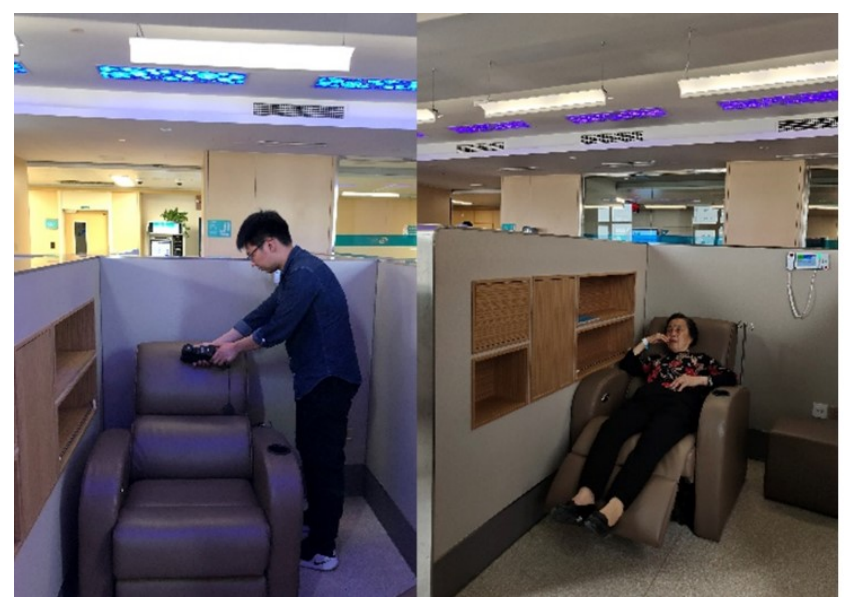

Figure 4-Photos of the experiment site

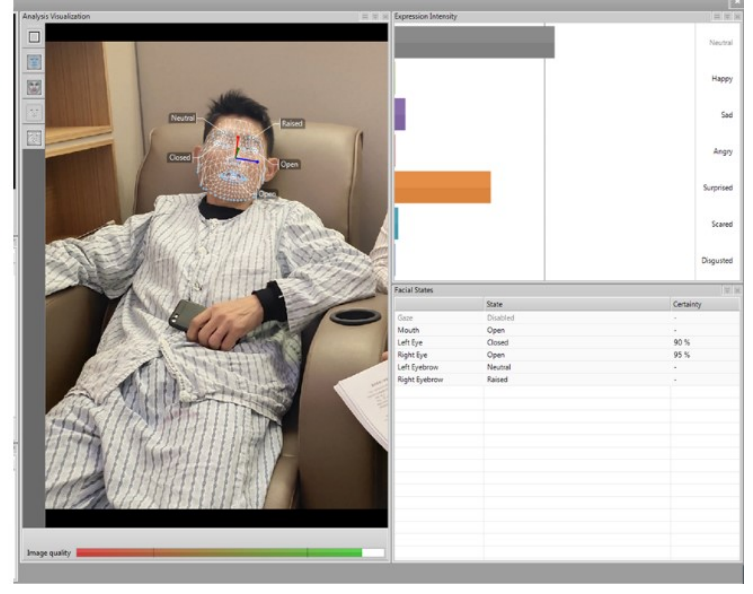

Figure 5 - Analysis interface for Facereader software

\subsection{Experimental process}

After the subjects adapted to different light environment modes for 1 hour, the photos of facial expressions were collected, and the subjects were not disturbed as much as possible. After showing all the scene patterns, the subjects were interviewed and the satisfaction survey was conducted (see figure 4).

\subsection{Analytical methods}

By using the Deep neural network algorithm built into FaceReader facial expression analysis software, the facial photos of subjects under different light environments are analysed (see figure 5). Then the percentage of subjects in different emotional states (happy, sad, afraid, disgusted, surprised, angry, neutral and others) is got. Based on the data of various basic emotions, the total percentage of negative emotions (sadness, fear, disgust, surprise and anger) can be obtained. Finally, by comparing the percentage of negative emotions of the subjects under different light modes, the appropriate light mode was obtained.

\section{Experimental results and discussion}

\subsection{Gender difference}

First, all the subjects were statistically analysed according to gender, and the average percentages of negative, positive, neutral and other emotions of male and female subjects were calculated respectively, with no significant difference (see figure 6). Then, subjects in the reference group and the three intervention groups were statistically analysed by gender, and their mean values were taken as the emotional characteristic values of the group (see figure 7).There was no significant gender difference between the control group and the purple group. The percentage of partial emotions in the orange group and the blue group was significantly different, mainly reflecting the positive emotions in the orange group and the negative emotions in the blue group. Considering individual differences, more sample sizes are needed to illustrate this issue in subsequent studies. 


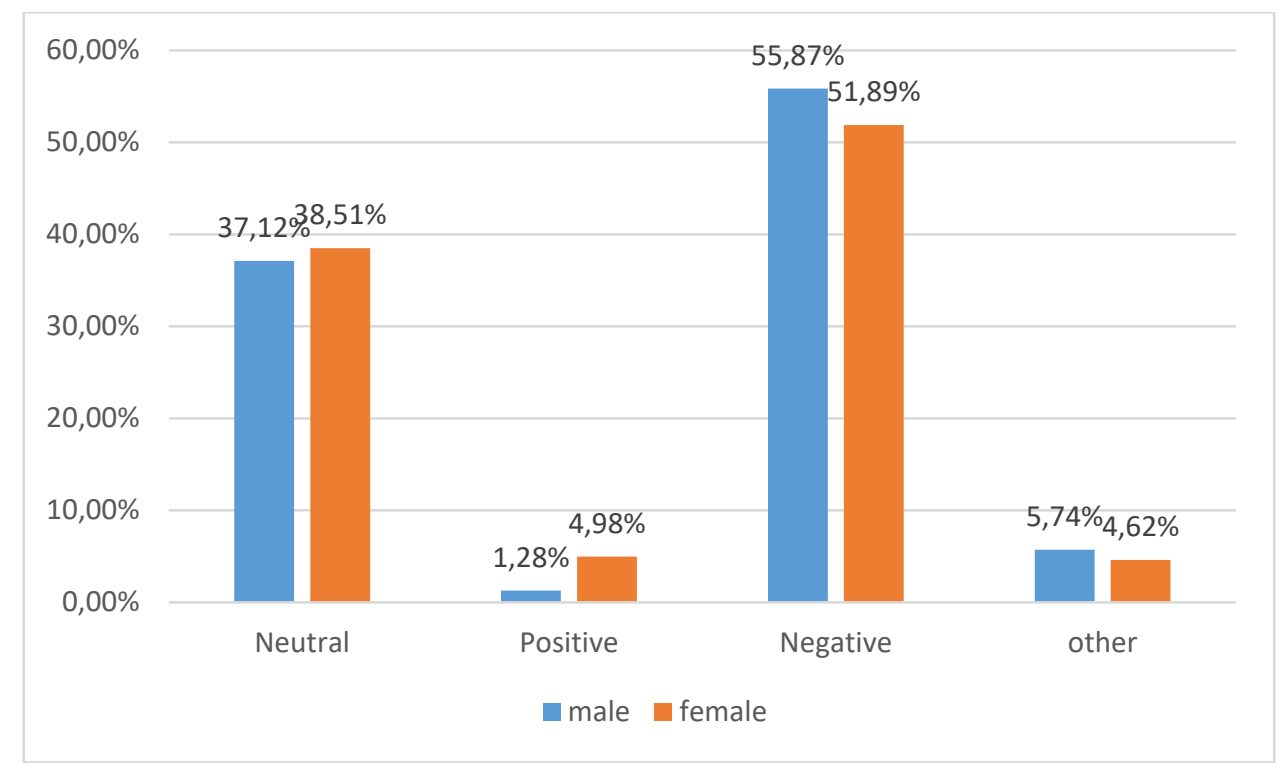

Figure 6 - Gender differences in emotions of all subjects

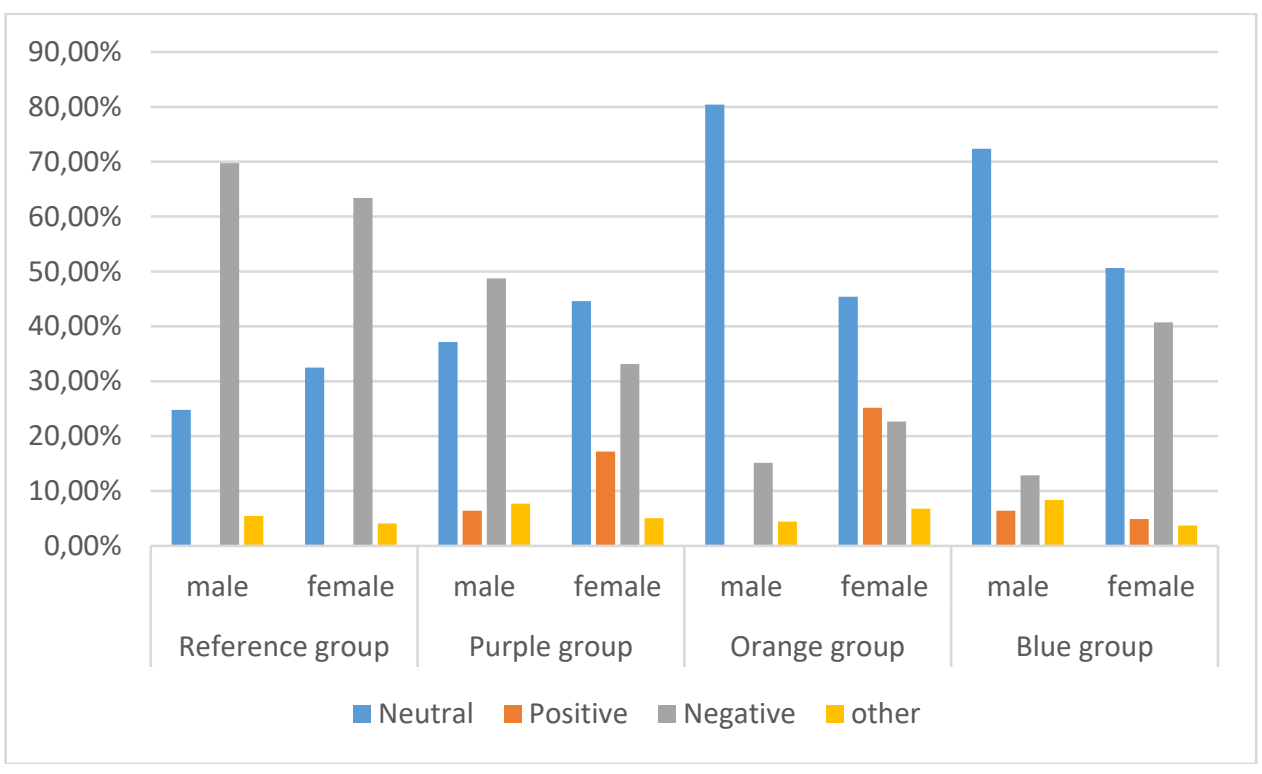

Figure 7 - Gender differences in emotions of subjects in different groups

\subsection{Colour difference}

First, the basic emotions of each group were analysed, and the average value was taken as the representative value of the basic emotions of the group (see figure 8 ). In the control group, the percentage of sadness was as high as $66.58 \%$ without the intervention of mood panel lights. The sadness of the experimental group did not exceed $20 \%$, among which the orange group was the lowest, only $3.40 \%$. The control group had the lowest percentage of happy mood, followed by the blue group $(5.65 \%)$, and the orange group and the purple group had higher percentage of happy mood. The control group had the lowest neutral emotions, followed by the purple group, the orange group and the blue group. The control group and the blue group had lower surprise, while the orange group and the purple group had higher surprise. The purple group had the highest fear, while the orange group and the blue group had the lowest fear. There was no significant difference between the other mood groups. 


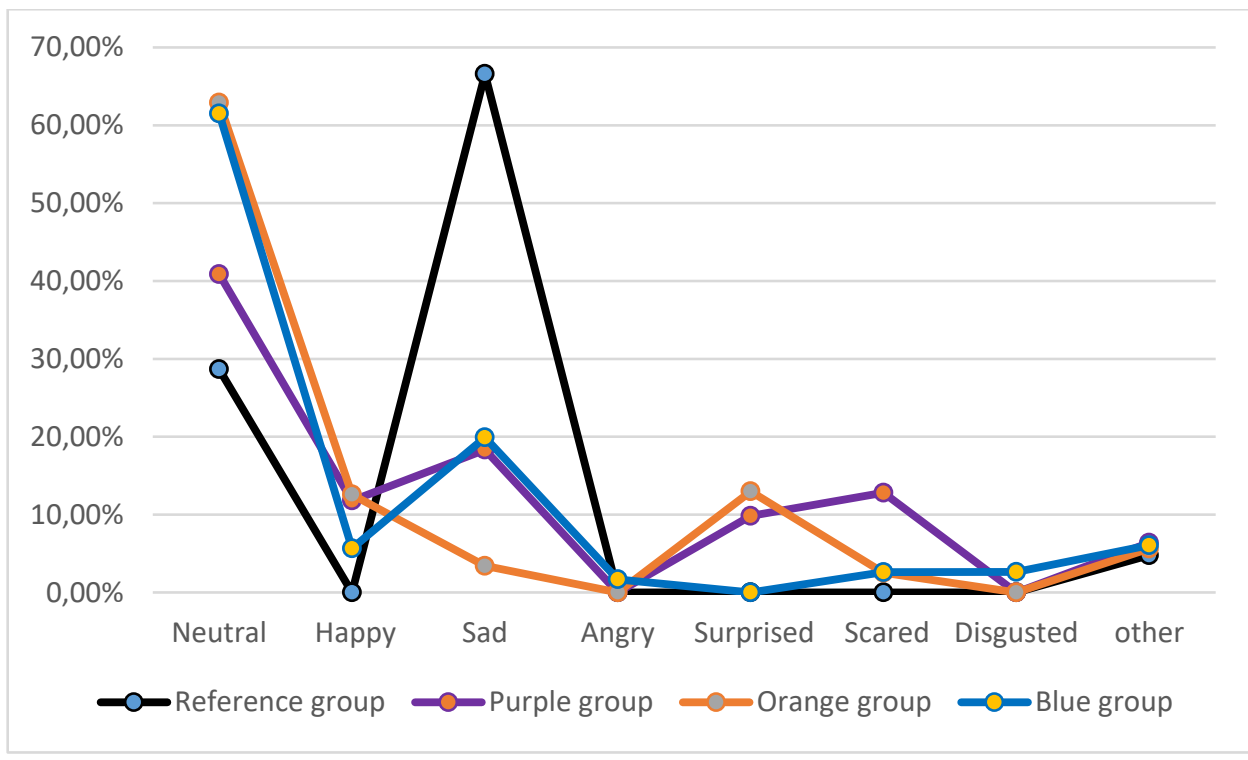

Figure 8 - Changes of basic emotions of subjects in different groups

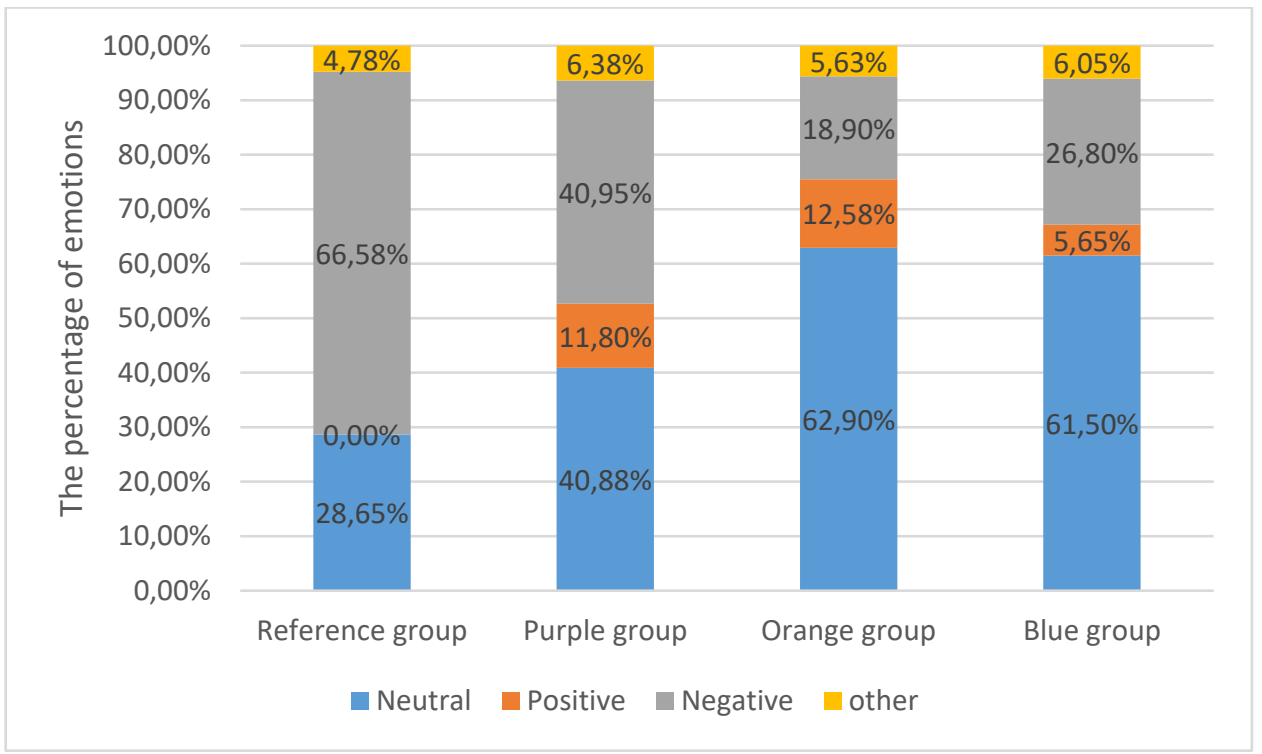

Figure 9 - Percentage of main emotions of subjects in different groups

On the basis of basic emotional analysis, negative emotions, positive emotions, neutral emotions and other emotions of each group were summarized (see figure 9). For negative emotions, the proportion of the reference group was the highest, reaching $66.58 \%$, followed by the purple group $(40.95 \%)$, and the proportion of negative emotions in the blue group and the orange group was lower. For positive emotion, the proportion of the reference group was the lowest, the blue group (5.65\%) was lower, the orange group $(12.58 \%)$ and the purple group $(11.80 \%)$ were higher. For neutral (natural) emotions, the orange group had the highest proportion, followed by the blue group $(61.50 \%)$, and the reference group had the lowest proportion.

\subsection{Subjective preference of colour}

In view of the existing four colour modes of mood panel lights (purple, orange, blue and cyan), a live demonstration and a questionnaire survey were conducted. It is found that patients with low vision are not satisfied with the existing several scene modes, and they hope to have more colour choices, especially the preference for green is very high, and some people feel indifferent (see figure 10).During the interview, many patients mentioned that they should control the 
colour of emotional lights instead of the existing preset mode to enhance the interactive experience, which should be considered in the subsequent scene design.

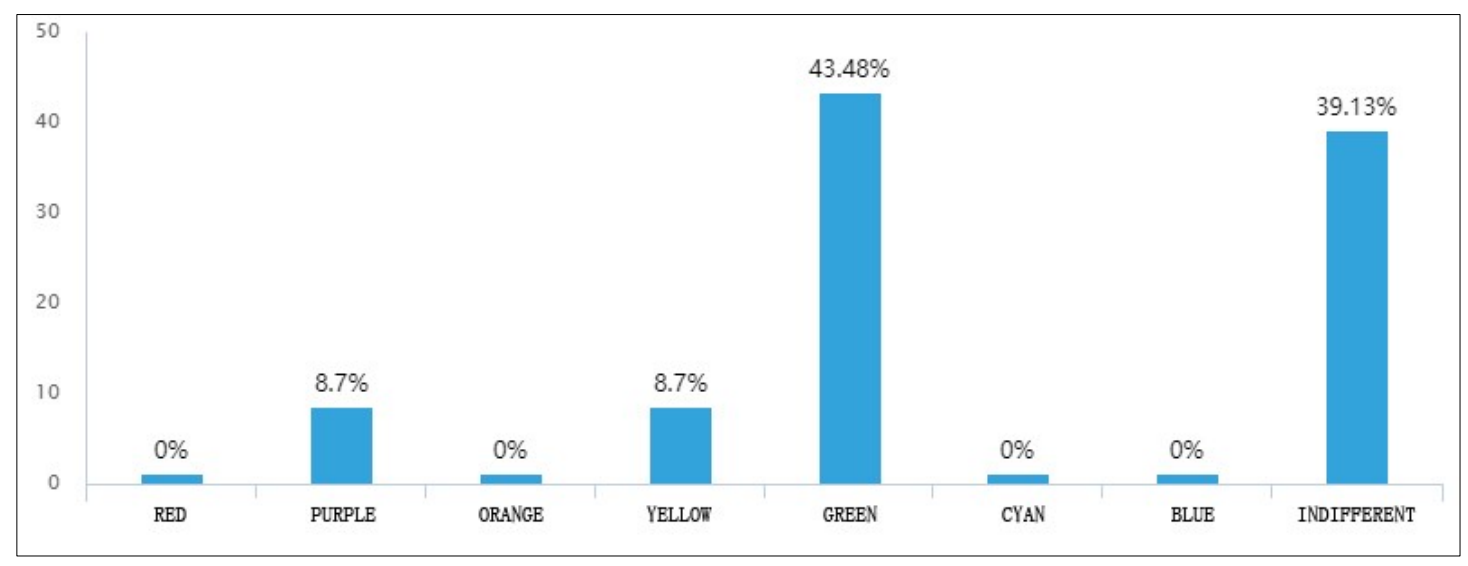

Figure 10 - Patients' subjective preference for colour of emotional lights

\section{Conclusion}

Facial recognition software can effectively analyse the effect of light stimulation on low visual emotion, and indirectly judge the change of negative emotions of the subjects through the analysis of basic emotions. Light environment intervention can effectively reduce the negative emotions of patients with low vision and improve the treatment effect, but the specific light environment parameters (ambient brightness and ambient light colour) still need to be further studied.

\section{Funding}

National Key Research and Development Program of China (No. 2017YFB0403704).

\section{References}

CIE 2017. CIE 227:2017. Lighting for Older People and People with Visual Impairment in Buildings. Vienna: CIE.

Duan, Y. N. (2018). Quality of Life and its Influencing Factors in Adults with Low Vision. Cardiovascular Disease Journal of integrated traditional Chinese and Western Medicine, 6(15), pp.158.

Figueiro, M., Hunter, C., Higgins, P., Hornick, T., Jones, G., Plitnick, B., Brons, J. and Rea, M. (2015). Tailored lighting intervention for persons with dementia and caregivers living at home. Sleep Health, 1(4), pp.322-330.

Gao, Q. Q., Yang, R., Lv, C.L. (2009). Relationship among Psychological Health, Social Support and Coping Styles in Patients with Low-vision. Chinese Mental Health Journal,23(4), pp.242-245.

Ham, J.J., Wan, S., Lakens, D.D. (2012). The Influence of Lighting Color and Dynamics on Atmosphere. Perception and Relaxation.

Hao, L.X., Cao, Y.X., Cui, Z., Zeng, K., Shao, R.D. (2017). Research Trends and Application Prospects on Light and Health. China Illuminating Engineering Journal,28(06), pp.1-15+23.

Hatfield, N. and Kincheloe, C. (2013). Introductory maternity \& pediatric nursing.

Li, X.Y., Zhong, L.L., Huang, W.M. (2016). Effects of Psychological Intervention on Anxiety and Depression in Patients with Primary Glaucoma and Low Vision. Women's Health Research,09, pp. 70+68. 
Liu, W.Z. (2016). A Study on the Relationship Between Mental Health Status and Social Support in Patients with Low Vision. Clinical Journal of Chinese Medicine,8(16), pp.108-109.

Reed, L., Sayette, M. and Cohn, J. (2007). Impact of depression on response to comedy: A dynamic facial coding analysis. Journal of Abnormal Psychology, 116(4), pp.804-809.

Zhang, Q.W., Hu, Y.K., Weng, J., Peng, L., Chen, K.J., Wang, Y.F. (2018). Research on Lighting of Middle Section of Highway Tunnel Based on Psychological and Physiological Effects: Improving Driving Safety by Using Induced Lighting Devices (Retroreflective Materials). Light \& Lighting, 42(03),pp.1-9. 